\title{
Evolución de las lesiones por accidentes de trabajo y enfermedades profesionales en la República Argentina, 1997-2015
}

\author{
Maximiliano Rodríguez Giménezz, Monica Ubalde-Lopez,c, \\ Cecilia Cornelio ${ }^{d}$, Maria López-Ruiz ${ }^{a, b, c, e}$ \\ y Fernando G. Benavides ${ }^{a, b, c}$
}

DOI: 10.12961/aprl.2019.22.01.4

Recibido: 21 de septiembre de 2018

Aceptado: 10 de diciembre de 2018

\section{RESUMEN}

Objetivo: Describir la tendencia temporal de las lesiones por accidentes de trabajo (LAT) y las enfermedades profesionales (EP) en Argentina, entre 1997 y 2015, según gravedad del daño, lugar de ocurrencia, actividad económica y provincia.

Métodos: La población de estudio está formada por la población trabajadora asalariada cubierta por el sistema de Riesgos del Trabajo de Argentina, que era de aproximadamente 4 millones en 1997 y de 9.6 millones en 2015. La tasa anual de incidencia de las LAT y EP se calculó según el número de casos notificados dividido por el promedio de trabajadores cubiertos por el sistema en cada año. Para medir el cambio temporal en la incidencia se calculó el porcentaje de cambio general (PCG), como el cociente de la diferencia entre tasa del primer año menos la del último año, dividido por la tasa del último año.

Resultados: La incidencia global de LAT y EP disminuyó de manera más pronunciada entre los mortales (PCG = -65,0\%) que en los no mortales $(\mathrm{PCG}=-23,3 \%)$. Según el lugar de ocurrencia, se observó un descenso en la incidencia de las LAT no mortales en jornada $(\mathrm{PCG}=-47,7 \%)$ y un incremento de las in itinere $(\mathrm{PCG}=123,4 \%)$. En relación a las EP, se apreció un aumento en la incidencia de las EP no mortales (PCG=393,0\%). Las EP mortales mostraron una tendencia más oscilante. Estas tendencias se reproducen de manera similar tanto para las actividades económicas como para las provincias seleccionadas.

Conclusiones: Las tendencias observadas, especialmente en los últimos años, en las LAT y las EP en Argentina muestran un patrón similar al de países europeos, con un descenso de las LAT en jornada, mortales y no mortales, excepto para las LAT in itinere. Sin embargo, en el estudio de las EP no mortales se debe profundizar en el conocimiento más detallado y completo de su tendencia temporal.

PALABRAS CLAVE: salud ocupacional, lesiones por accidentes de trabajo, enfermedades profesionales, prevención.

TRENDS IN OCCUPATIONAL INJURY AND DISEASE IN ARGENTINA, 1997-2015

\section{ABSTRACT}

Objectives: To describe temporal trends in occupational injuries (OI) and occupational diseases (OD) in Argentina, by severity of the injury, place of occurrence, economic activity and selected provinces, in the period 1997 to 2015.

Methods: The study population consists of the working population covered by the Argentine Law of Occupational Risks system, roughly 4 million people in 1997 and 9.5 million in 2015. The annual incidence rate of the OI and OD was calculated by using the number of reported cases divided by the mean number of employees covered by the system each year. To measure change in incidence over time, we calculated the overall percentage change (OPC), by dividing the difference between the incidence rate of the first minus the last year by the incidence rate of the last year.

Results: The overall incidence of $\mathrm{OI}$ and $\mathrm{OD}$ showed a greater decline for fatal $(\mathrm{OPC}=-65 \%)$ versus nonfatal injuries (OPC-23,3\%). By place of occurrence, we observed a decrease in the incidence of nonfatal occupational injuries at the workplace (OPC $=-47,7 \%)$, but an increase in occupational commuting $\mathrm{OI}(\mathrm{OPC}=+123,4 \%)$. For $\mathrm{OD}$, there was an increase in the incidence of nonfatal $\mathrm{OD}(\mathrm{OPC}=393 \%)$,

a. Centro de Investigación en Salud Laboral (CISAL), Universidad Pompeu Fabra. IMIM Parc Salut Mar. Barcelona, España.

b. CIBER en Epidemiología y Salud Pública (CIBERESP), Madrid, España.

c. Instituto Hospital del Mar de Investigaciones Médicas (IMIM),

Barcelona, España.

d. Superintendencia de Riesgos del Trabajo (SRT), Ministerio de Trabajo,

Empleo y Seguridad Social, Buenos Aires, Argentina.

e. Facultad Latinoamericana de Ciencias Sociales (FLACSO),

Salamanca, España.
Correspondencia:

Mònica Ubalde Lopez

monica.ubalde@upf.edu

Centro de Investigación en Salud Laboral, Universidad Pompeu Fabra.

Carrer del Dr. Aiguader 88

08003 Barcelona, España. 
although the tendency showed both increases and declines among fatal occupational diseases. This pattern was consistent among different economic activities as well as in selected provinces.

Conclusions: Observed trends are in line with other high-income countries, with decreasing nonfatal workplace OI, and increasing commuting OI and nonfatal OD. Further in-depth research is warranted on temporal trends in non-fatal OD.

KEY WORDS: temporal trends, occupational injuries, occupational diseases, prevention.

\section{INTRODUCCIÓN}

Las lesiones por accidentes de trabajo (LAT) y las enfermedades profesionales (EP) continúan representando uno de los principales problemas en salud ocupacional en el mundo, aunque existen grandes diferencias entre las distintas regiones ${ }^{1}$. Según la Organización Internacional de Trabajo (OIT), cada día se producen 6.300 muertes a causa de accidentes o enfermedades relacionadas con el trabajo remunerado, lo que contabiliza más de 2,3 millones de fallecimientos al año en todo el mundo. Específicamente, se estima que cada año ocurren más de 317 millones de accidentes de trabajo, lo que supone que cada 15 segundos 153 personas que trabajan sufren un accidente laboral, siendo muchos de éstos lesiones con baja laboral². En 2007 la Organización Panamericana de la Salud estimó que en la Región de las Américas ocurrieron al menos 7,6 millones de accidentes de trabajo, siendo más frecuentes en hombres, los que causaron cerca de 11.343 muertes $^{3}$. Estas cifras pueden no reflejar el panorama regional completo, ya que no se incluye el amplio sector informal de la región, además de la existencia de un elevado subregistro en cada país, especialmente en relación con las enfermedades profesionales $^{4,5}$.

En Argentina, a finales de 1995 entra en vigor la Ley de Riesgos del Trabajo (en adelante LRT) y se establece el marco normativo que recoge la obligatoriedad por parte de las personas empleadoras, tanto de asegurar a la población trabajadora frente a los riesgos laborales, así como las definiciones y los sistemas de notificación y el registro de las LAT y EP ${ }^{6,7}$. La información recogida en los registros durante los siguientes 20 años hace posible dar una visión detallada de la tendencia temporal de la incidencia de las LAT y EP según gravedad del daño y lugar de ocurrencia o llevado acabo anteriormente. El objetivo del presente trabajo fue describir la tendencia de las lesiones por accidentes de trabajo y las enfermedades profesionales de la República Argentina entre los años 1997 y 2015, según la gravedad del daño, el lugar de ocurrencia, la actividad económica y por provincias seleccionadas.

\section{MÉTODOS}

Estudio descriptivo de tendencia temporal de la tasa de incidencia de las LAT y las EP durante el período comprendido entre los años 1997 y 2015. La población de estudio está formada por la población trabajadora cubierta por el sistema de riesgos del trabajo de Argentina, gestionada a través de las Aseguradoras de Riesgos del Trabajo (ART), como son las personas asalariadas públicas y privadas, y quienes trabajan de manera remunerada en casas particulares. En su conjunto, para el periodo de estudio, la población cubierta ha pasado de 4.124 .726 en 1997 a 9.674 .906

Arch Prev Riesgos Labor 2019; 22 (1): $18-24$ en $2015^{8}$. Los datos utilizados en el presente estudio provienen de informes y enlaces electrónicos de la Superintendencia de Riesgos del Trabajo (SRT) ${ }^{9}$.

Las variables principales del estudio son las LAT y las EP, que fueron sumadas para cada año, teniendo en cuenta las reagravaciones que incluye un nuevo caso, ya que no fue posible separarlas. Además, se consideraron como variables de estratificación: 1) la gravedad del daño: mortales y no mortales; 2) el lugar de ocurrencia: en jornada de trabajo o in itinere. Es decir, las que ocurren durante la jornada laboral habitual, o en el trayecto entre el domicilio del trabajador y su lugar de trabajo, respectivamente; 3) la actividad económica, según la Clasificación Industrial Internacional Uniforme (CIIU): agricultura, minería, manufacturas, electricidad, construcción, comercio, transporte, servicios financieros y servicios sociales, y 4) provincias seleccionadas: Ciudad Autónoma de Buenos Aires, Provincia de Buenos Aires, Provincia de Córdoba y Provincia de Santa Fe, que representan en conjunto más del 70\% de la población cubierta por el sistema.

Respecto al análisis, la tasa de incidencia anual de las LAT y EP fue calculada según el número total de casos notificados dividido por el número promedio de personas trabajadoras cubiertas por el sistema en cada año multiplicado por 1.000 para las LAT y por 10.000 para las EP. Asimismo, las tendencias temporales desde 1997 a 2015 de LAT y EP según las variables descriptivas anteriormente mencionadas se representaron gráficamente para todos los años, cuando la información estuvo disponible.

Finalmente, para valorar la tendencia en el conjunto del periodo, o en subperiodos más cortos, se calculó el porcentaje de cambio general $(\mathrm{PCG}): \mathrm{PCG}=(\mathrm{It} 1-\mathrm{It} 0) / \mathrm{It} 0$ x 100; donde It 1 es el último año del periodo comparado e It0 el primer año de dicho periodo. Igualmente, se calculó el porcentaje de cambio promedio anual (PCPA), en aquellos periodos o subperiodos donde la tendencia se mantenía gráficamente estable: PCPA = [[(It1 - It0) / It0]/ n] x 100, donde $\mathrm{n}$ es el número de años entre t0 y t $1+1$.

\section{RESULTADOS}

La incidencia global de LAT y EP incluyendo en jornada, in itinere y reagravaciones muestra un descenso entre 1997 y 2015, que fue más pronunciado entre las mortales (PCG $=-65 \%$ ) que en las no mortales (PCG $=-23,3 \%$ ). En el caso de las LAT y EP no mortales -evaluadas conjuntamente- se observó un descenso inicial que se detiene en 2002 (62,14 por 1.000), incrementándose hasta 2005 (81,46 por 1.000$)$ y manteniéndose desde entonces estable hasta $2008(80,58$ por 1.000$)$, para luego volver a descender hasta 2015. Por el contrario, la tendencia descendente de las mortales fue constante, con un PCPA de $-3,4 \%$ (Figura 1). 
FIGURA 1

Incidencia anual de Lesiones por Accidente de Trabajo ${ }^{a}$ y Enfermedad Profesional (global), según la gravedad del daño.

Argentina, 1997-2015.

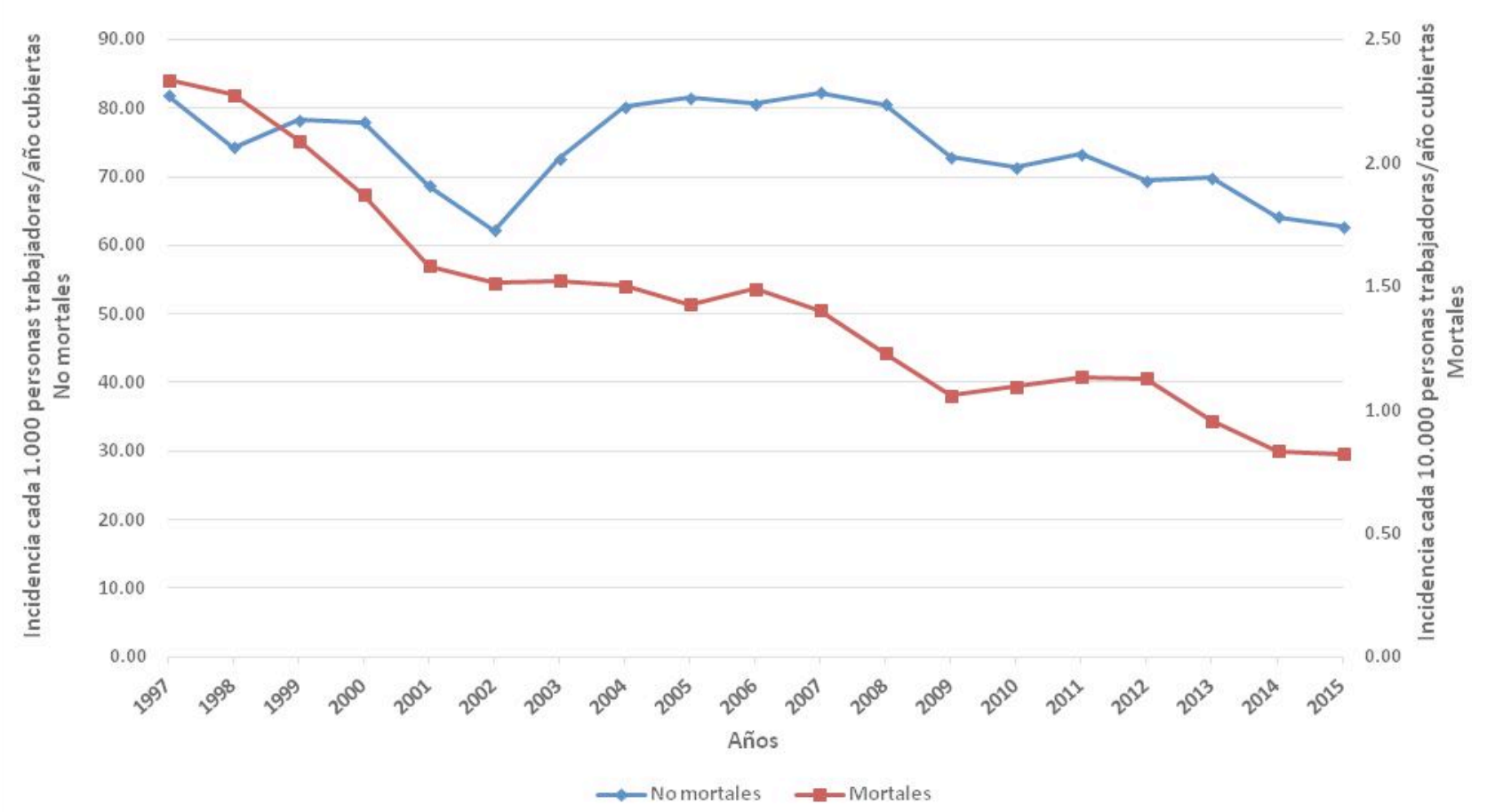

a. Incluye accidentes de trabajo en jornada, in itinere, y reagravaciones.

Al descomponer las LAT y EP no mortales según la actividad económica (Figura 2), se aprecia un descenso global en las mismas en el periodo de estudio. En el caso particular de la construcción, el descenso se detuvo en 2002 (pasando de 168,28 en 1997 a 107,78 por 1.000 en el 2002), incrementándose hasta 2006 (177,71 por 1.000), para volver a descender hasta 2015. Una tendencia similar se observó en la industria manufacturera, aunque con valores inferiores de la tasa de incidencia. En el resto de las actividades económicas se observa una tendencia estable, con leves descensos y una clara convergencia hacia el final del período estudiado.

La incidencia de las LAT y EP no mortales (incluyendo en jornada, in itinere y reagravaciones), analizada según las provincias seleccionadas (Figura 3), muestra un descenso en Ciudad Autónoma de Buenos Aires (C.A.B.A) (PCG = -32,2\%), Santa Fe $(\mathrm{PCG}=-25,1 \%)$ y Córdoba $(\mathrm{PCG}=-10,4 \%)$. Por el contrario, se aprecia un leve aumento en la provincia de Buenos Aires (PCG $=+6,6 \%$.

Al separar las LAT no mortales, según lugar de ocurrencia (Figura 4), se observa un descenso de las LAT no mortales en jornada (PCG $=-47,7 \%$ ), junto con un incremento de las in itinere (PCG $=123,4 \%$ ). En el caso de las LAT en jornada se apreció un descenso inicial que se detuvo en 2003 (61,02 por 1.000), incrementándose hasta el 2005 (69,03 por 1.000), tras lo cual vuelve a descender hasta 2015 (incidencia de 42,21 por 1.000). Por el contrario, la tendencia de las in itinere se ha incrementado de forma constante a lo largo del período estudiado, con un incremento de la PCPA del 6,5\% anual.
El análisis específico la incidencia de EP (Figura 5), muestra un aumento de aquellas no mortales, con y sin baja laboral, entre 1997 y 2009 donde alcanza su punto máximo de 39,44 casos por 10.000 (PCG $=843,5 \%$ ). Posteriormente, a partir de 2009, cambia la tendencia descendiendo hasta 2015 con una incidencia de 20,61 casos por $10.000(\mathrm{PCG}=-47.7 \%)$. Por el contrario, las EP mortales siguen una tendencia en dientes de sierra, con ascensos y descensos a lo largo de todo el período estudiado, y representan un número absoluto muy reducido.

\section{DISCUSIÓN}

Los resultados muestran que la evolución global de la incidencia de LAT y EP en Argentina entre 1997 y 2015, tanto para casos mortales como no mortales, muestra un claro descenso durante el periodo estudiado. Esta reducción se observa especialmente en las LAT no mortales que ocurren en jornada de trabajo. Por el contrario, en el análisis desagregado se aprecia un aumento en la incidencia de las LAT ocurridas in itinere y de las EP no mortales. Estas tendencias generales se reproducen de manera similar tanto en las diferentes actividades económicas, como en las provincias seleccionadas.

Los resultados respecto a las LAT no mortales en jornada concuerdan con aquellos observados en otros países como Espa$\tilde{n} a^{10}$, donde entre 2000 y 2009 el riesgo de sufrir LAT no mortal disminuyó en una proporción similar a la encontrada en Argentina. En España, el descenso fue del 46\% y en Argentina del 48\%, aunque este último en un período más prolongado (1997-2015). Un patrón similar se ha observado también para este mismo periodo en un grupo seleccionado de países de la Unión Europea ${ }^{11}$ y de EEUU ${ }^{12}$. 
Evolución de las lesiones por accidentes de trabajo y enfermedades profesionales en la República Argentina, 1997-2015

21

FIGURA 2

Incidencia anual de Lesiones por Accidente de Trabajo y Enfermedad Profesional (global), no mortales, según la actividad económica. Argentina, 1997-2015.

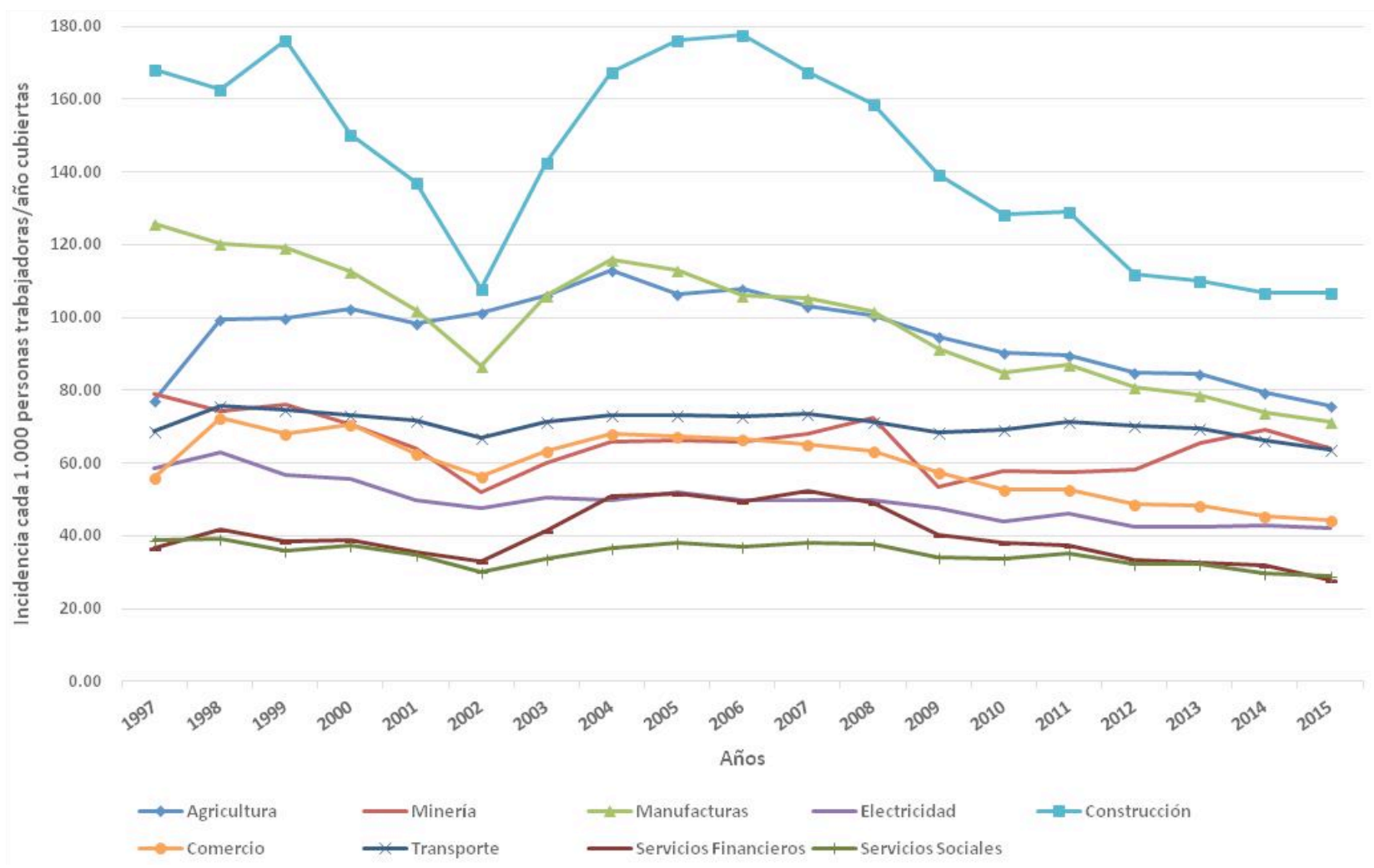

FIGURA 3

Incidencia anual de Lesiones por Accidente de Trabajo no mortales, según el lugar de ocurrencia. Argentina, 1997-2015.

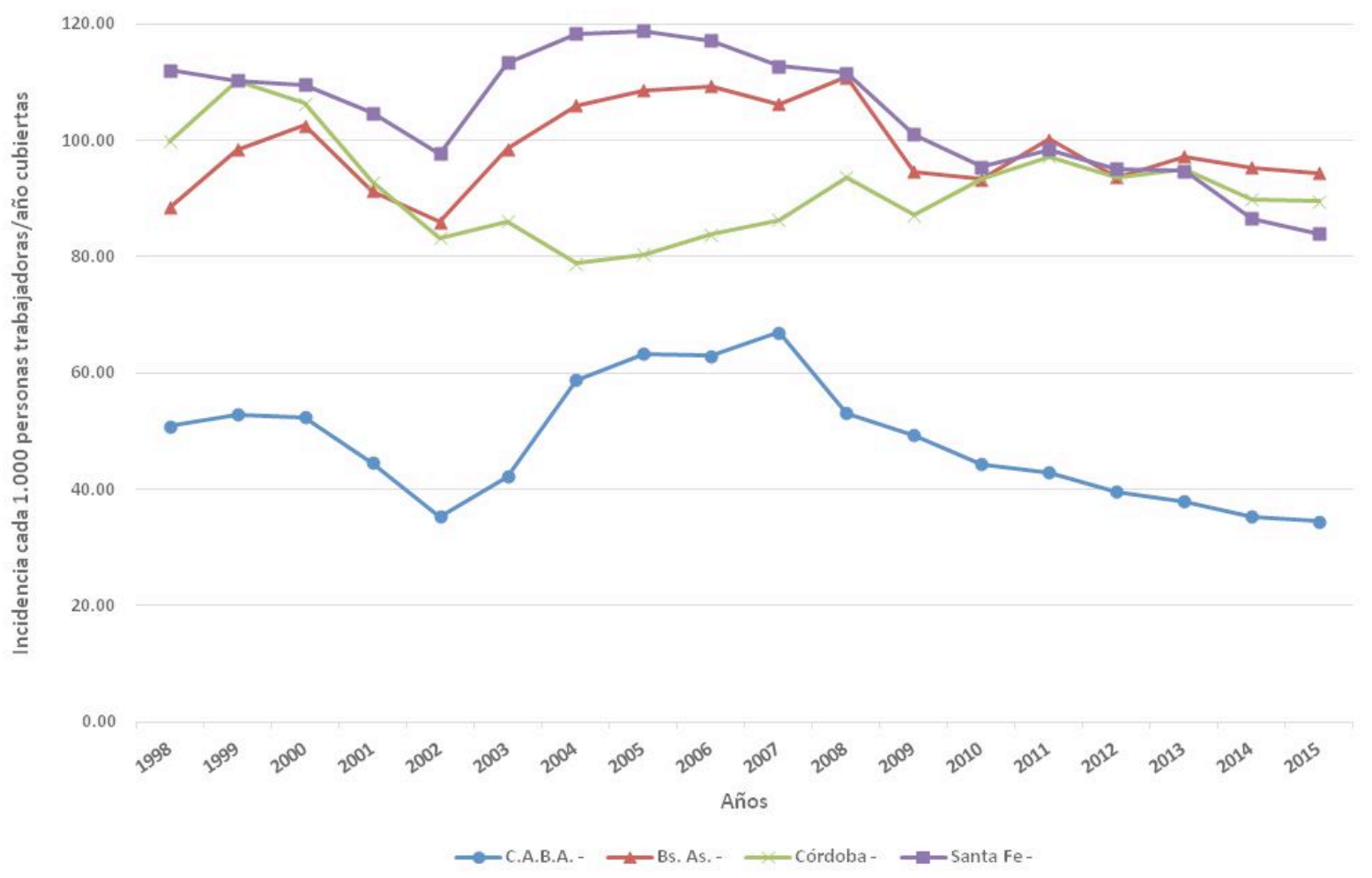

a. Incluye accidentes de trabajo en jornada, in itinere, y reagravaciones; C.A.B.A., Ciudad Autónoma de Buenos Aires.

Arch Prev Riesgos Labor 2019; 22 (1): 18-24 
Evolución de las lesiones por accidentes de trabajo y enfermedades profesionales en la República Argentina, 1997-2015

22

FIGURA 4

Incidencia anual de Lesiones por Accidente de Trabajo no mortales, según el lugar de ocurrencia. Argentina, 1997-2015.

90.00

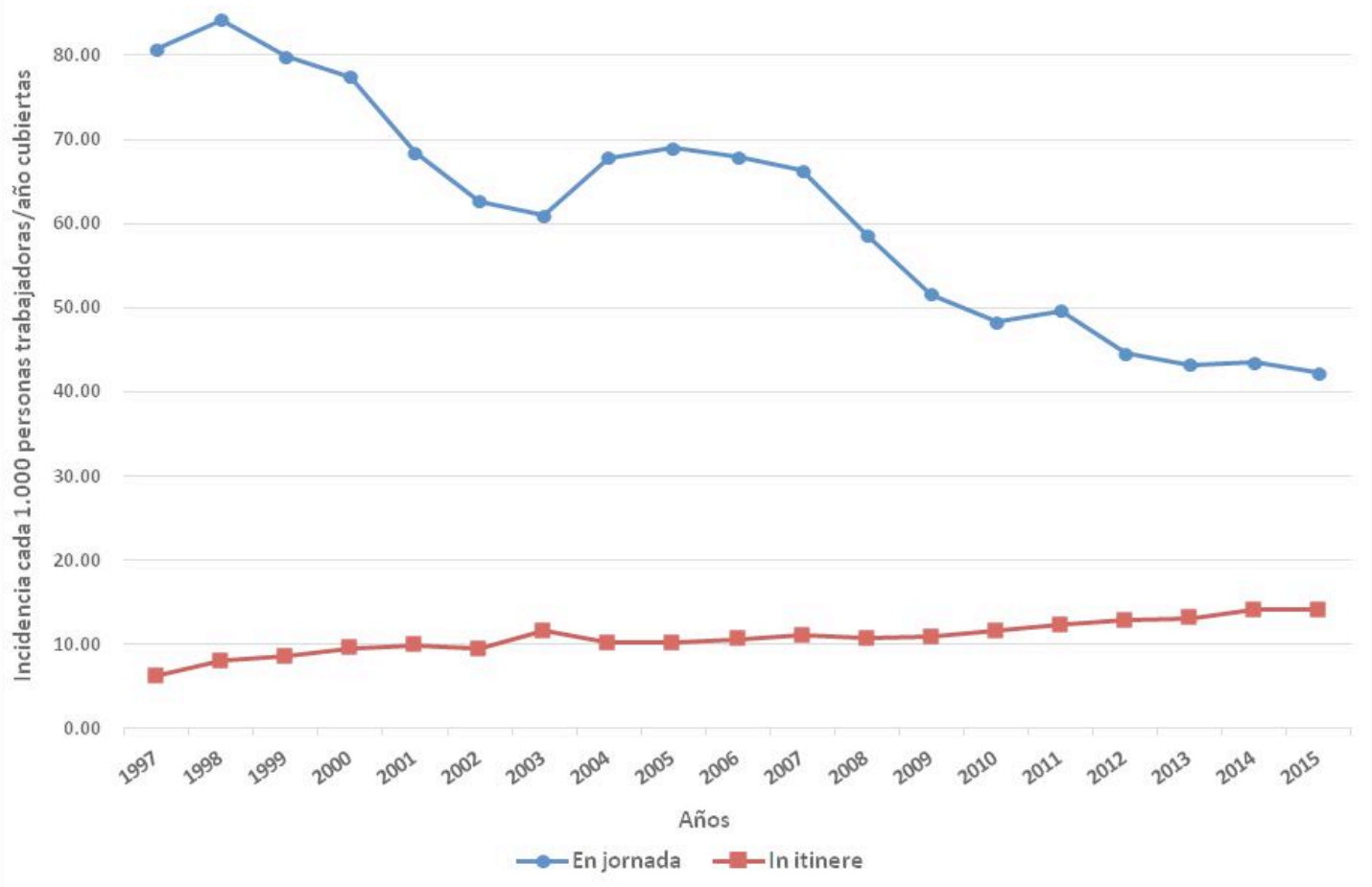

FIGURA 5

Incidencia anual de Enfermedad Profesional*. Argentina, 1997-2015.

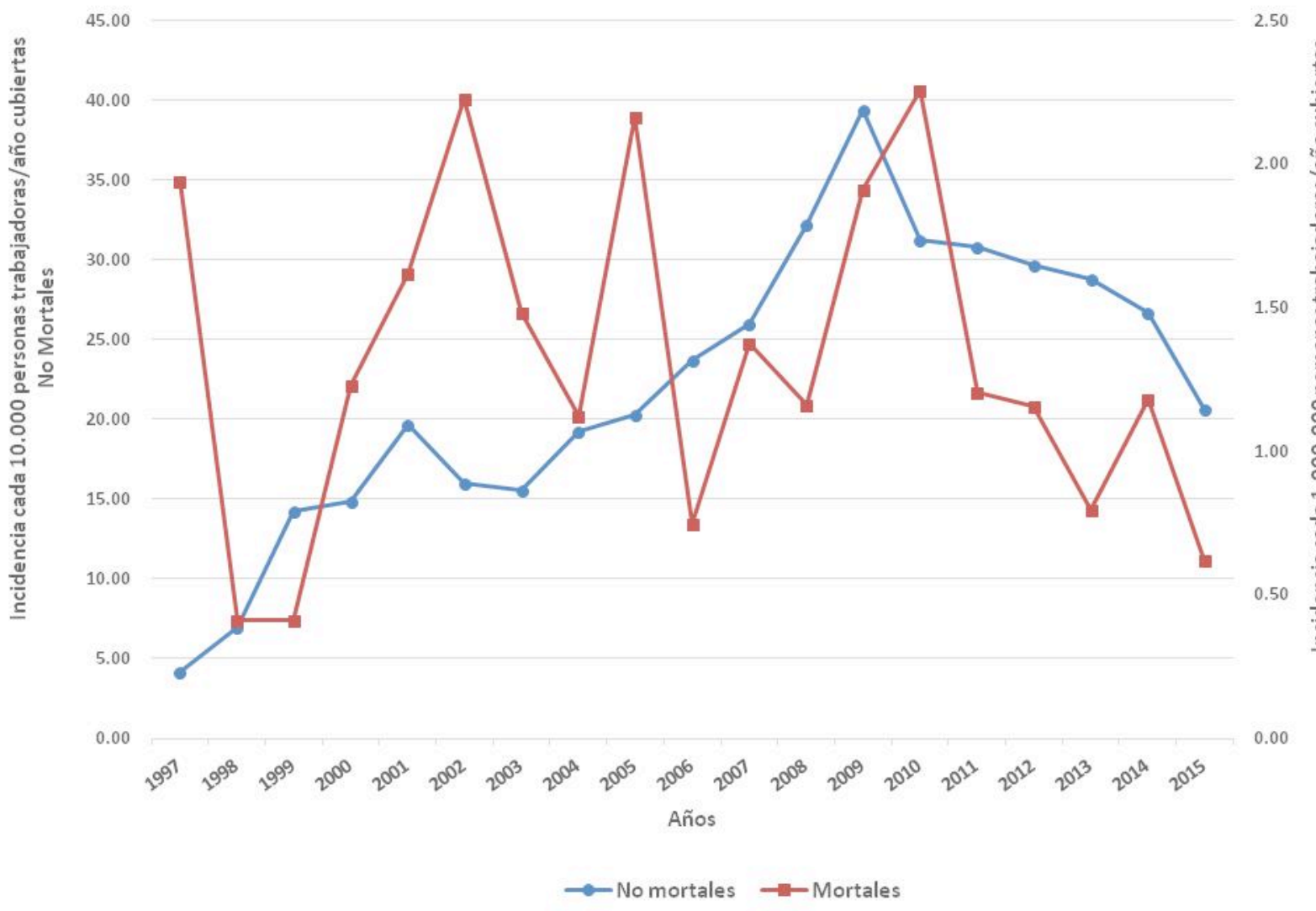

* Incluye Ep no mortales con y sin baja laboral.

Arch Prev Riesgos Labor 2019; 22 (1): 18-24 
Una primera hipótesis para explicar esta disminución global podría estar relacionada, al menos en parte, con la aprobación de la LRT en 1995, y el consiguiente esfuerzo de administraciones, empresas, empleadores, sindicatos y personas trabajadoras en llevar a cabo actividades preventivas de las LAT y EP. Esta hipótesis, sin embargo, no la podemos contrastar, ya que no es posible comparar con un periodo anterior a la entrada en vigor de la LRT, que nos serviría de control para valorar si hay un cambio significativo en la tendencia. Por otro lado, no podemos olvidar los cambios tecnológicos y organizativos introducidos en las empresas, que de manera indirecta también podrían haber mejorado significativamente las condiciones de trabajo, reduciendo la exposición a factores de riesgo. Estas hipótesis explicativas pueden estar más ajustadas a las tendencias de las LAT y EP mortales. Tal como se pudo observar en España, donde la incidencia de las LAT mortales en jornada laboral descendió un 38\% entre 1992 y 2002, pasando de 9,8 a 6,1 casos por 100.000 personas asalariadas, estimándose que los cambios en la estructura productiva (más servicios y menos agricultura e industria) podían explicar dos terceras partes de este descenso, el otro tercio se podría explicar por las intervenciones preventivas ${ }^{13}$. En un estudio similar realizado en Estados Unidos para el periodo 1980-1996, se observó un descenso de las LAT mortales fue del 45\%, dos terceras partes atribuibles a las actividades preventivas ${ }^{14}$.

De manera más particular, para las LAT no mortales se observa de manera consistente un punto de inflexión en el 2002 tanto en la incidencia global como por actividades económicas y provincias. La posible explicación de esta tendencia decreciente de las LAT no mortales en jornada de trabajo en Argentina entre 1997-2002, creciente a partir de entonces hasta 2006 y con un nuevo descenso hasta 2015, podría estar relacionada con dos factores: i) la crisis económica profunda que afectó severamente a la Argentina de manera dramática entre 2000-2002 y ii) la recuperación económica, a partir del 2006, que permitió introducir mejoras en la estructura productiva así como incorporar actividades preventivas. Unas hipótesis que requieren de futuros estudios para su verificación.

Por el contrario, en relación con las LAT no mortales in itinere se aprecia un incremento continuado a lo largo de todo el período estudiado. Un fenómeno diferente ocurre en España, donde las LAT no mortales in itinere entre 2004 y 2010 han seguido un comportamiento distinto, con una tendencia descendente (se pasó de 81.404 casos en el año 2004 a 74.885 en 2010), lo que representa una disminución global del $-8 \%{ }^{15}$.

En el caso particular de Argentina, este fenómeno podría estar explicado, en primer lugar, al incremento del parque vehicular ${ }^{15}$, tanto de automóviles como de motocicletas, así como de los cambios en las estructuras urbanas que incrementan las distancias que una persona debe recorrer para llegar a su lugar de trabajo desde su domicilio habitual, lo que podría postularse también como un potencial factor que aumentaría el riesgo de LAT in itinere. De hecho, el mayor riesgo de LAT in itinere se observa en la provincia de Buenos Aires con respecto al resto de provincias analizadas. El 47,7\% de los puestos de trabajo existentes en C.A.B.A son ocupados por personas que residen en el conurbano

Arch Prev Riesgos Labor 2019; 22 (1): $18-24$ bonaerense (que forma parte de la Provincia de Buenos Aires), por lo que están obligadas a recorrer grandes distancias diariamente hasta llegar a su lugar de trabajo, aumentando el riesgo de sufrir una lesión en su traslado ${ }^{16,17}$. De nuevo, sería necesario realizar futuros estudios que verifiquen si esta hipótesis es cierta.

Con respecto a las EP mortales, lo primero a destacar es el bajo número de casos, lo que dificulta un análisis de su tendencia temporal, que podría ser debido a una probable infra notificación, tal como ocurre en la mayoría de los países del mundo ${ }^{18,19}$. En este sentido, se debería comprobar si efectivamente en Argentina estamos también sufriendo de este problema secular de la infra-notificación y subregistro de las EP.

Respecto a la tendencia de las enfermedades profesionales no mortales, el aumento progresivo que se observa hasta el año 2008 puede tener su origen en el posible aumento real en la incidencia de nuevos casos de EP. Sin embargo, no se puede descartar que contenga un número de casos crecientes de origen laboral incierto o no específicos. Igualmente, esta tendencia creciente condujo en los años 2007-2009 a un incremento de los procesos de validación para el reconocimiento de una EP, lo que podría explicar el descenso observado posteriormente. Por todo ello, es necesario seguir profundizando en la investigación de las EP no mortales, de cara a tener una visión más detallada y completa de su tendencia temporal.

Este estudio, tiene limitaciones que es necesario considerar. En primer lugar, cabe señalar la utilización de datos agregados, pues no fue posible acceder a los datos individualizados (microdatos). Así, en algunos casos no fue posible separar entre las LAT y las EP, así como entre aquellas con baja de las sin baja laboral, o entre las LAT en jornada laboral de las in itinere, lo que dificulta la interpretación de algunas tendencias. Asimismo, tampoco fue posible, en algunas ocasiones, descontar las reagravaciones de las LAT. Por otro lado, tampoco fue posible desagregar por provincia de ocurrencia, lo que nos llevó a seleccionar aquellas que representan más del 70\% de la población trabajadora cubierta por el sistema de riesgos del trabajo de Argentina. Igualmente, el análisis desagregado por actividad económica no permitió el análisis específico de la categoría "trabajadoras/es de casas particulares", al no disponer de datos de toda la serie temporal estudiada, así como la categoría "no clasificada" por incluir menos de un $1 \%$ de los casos. Asimismo, la no disponibilidad de los datos desagregados por sexo no hizo posible valorar las diferentes tendencias en hombres y mujeres. Finalmente, el presente estudio no pudo incluir a las personas trabajadoras ocupadas en empleo informal; es decir, sin aportes jubilatorios y sin prestaciones laborales. Tampoco se incluyeron personas trabajadoras por cuenta propia, ya que no tienen la obligación de contratar los servicios de una ART, por lo que están fuera de los registros de la SRT. En relación con estas dos últimas limitaciones, hay que señalar que aproximadamente la mitad de la población trabajadora de Argentina queda fuera del alcance del estudio, pues del total de la población ocupada, se estima que alrededor del 34\% trabaja con empleos informales (para la que no existen registros), y cerca del $20 \%$ es cuentapropista ${ }^{20}$, es decir pertenecen a población trabajadora sin cobertura por la SRT.

A pesar de dichas limitaciones, el estudio cuenta con relevantes fortalezas. Hasta donde sabemos, este es el primer trabajo 
que analiza la tendencia temporal de las lesiones por accidente de trabajo y enfermedades profesionales en Argentina, en un periodo de casi 20 años. Además, el acceso a los datos disponibles ha permitido reconstruir series temporales sobre las LAT y EP, para todos los sectores económicos y las provincias más representativas, de la población cubierta por el sistema de la LRT de la República Argentina.

En conclusión, si bien en Argentina existe un volumen muy elevado de población ocupada con empleo informal o cuentapropista de la que no conocemos la incidencia de LAT ni de EP, podemos confirmar que la tendencia secular decreciente de los daños a la salud de la población trabajadora argentina es similar a la que se observa en otros países, excepto para las LAT in itinere y las EP no mortales que se incrementan.

\section{AGRADECIMIENTOS}

A mi familia que me apoyó en todo momento: Mario Tomás Sasso, María José Gelpi, Norberto Rodríguez Giménez, Nicolás Rodríguez Giménez, Manuela Rodríguez Giménez y Silvina Beatriz Sasso.

A las compañeras del CiSAL por la ayuda prestada durante el desarrollo de este trabajo: Laura Serra Saurina, Montse Fernández Busquets.

A mis amigos y amigas que colaboraron conmigo en diferentes oportunidades: Julio César Hernando Rodríguez, Giovanny Michael Silva Peñaherrera, Carmen Luz Salas Quijada, Andrew March, Rocio Villar Vinuesa.

\section{LABORALIA 2019: \\ CERTAMEN INTEGRAL DE LA PREVENCIÓN \\ Y EL BIENESTAR LABORAL}

27-28 de marzo de 2019, Valencia

Información:

Web: www.laboralia.es/

\section{BÚSQUEDA E IMPLEMENTACIÓN \\ DE EVIDENCIA CIENTÍFICA: \\ UN ELEMENTO CENTRAL EN LA EXPERIENCIA DE OSH}

2.4 de abril de 2019, Reikiavik (Islandia)

\section{Información:}

Erika Gustafsson-Sundquist, NIVA, Arinatie 3A,

FI-00370 Helsinki, Finlandia.

Tel: +358405795787

E-mail: erika.gustafsson@niva.org

Web: https://niva.org/course/finding-and-implementingscientific-evidence-a-core-element-in-osh-expertise/

\section{BIBLIOGRAFIA}

1. Yanhong Ma, Qiuhong Zhao. Decision-making in safety efforts: Role of the government in reducing the probability of workplace accidents in China. Saf Sci. 2018; 104:81-90.

2. OIT, Seguridad y salud en el trabajo A: OIT. Organización Internacional del Trabajo [en línea]. Suiza, Ginebra: OIT [citado 01 Sep 2018]. Disponible en: http://www.ilo.org/global/topics/safety-and-health-at-work/lang--es/index.htm. 3. Organización Panamericana de la Salud. Salud en las Américas 2012: Panorama regional y perfiles de país. Washington, D.C.: OPS; 2012 [citado 01 Sep 2018]. Disponible en: http://www1.paho.org/saludenlasamericas/docs/sa2012-resumen.pdf.

4. Concha BM, Labbé J. Enfermedades profesionales: una aproximación a su frecuencia. Ciencia \& Trabajo 2007;9:117-120.

5. Organización Internacional del Trabajo. La prevención de las enfermedades profesionales. Ginebra: OIT; 2013 [citado 01 Sep 2018] Disponible en: http:// www.ilo.org/wcmsp5/groups/public/_ed_protect/-protrav/_safework/documents/publication/wcms_209555.pdf.

6. Ley sobre Riesgos del Trabajo. L. No 24.557/95 (13 Sept 1995).

7. Decreto sobre Riesgos del Trabajo. D. N 658/96 (24 Jun 1996).

8. SRT, Departamento de estudios y estadística A: SRT. Superintendencia de Riesgos del Trabajo [página principal en internet]. República Argentina: SRT [citado 01 Sep 2018]. Disponible en: https://www.srt.gob.ar/index.php/estadisticas-srt/serie-historica/.

9. SRT, Departamento de estudios y estadística A: SRT. Superintendencia de Riesgos del Trabajo [página principal en internet]. República Argentina: SRT [citado 01 Sep 2018]. Disponible en: https://www.srt.gob.ar/index.php/estadisticas-srt/accidentabilidad-ultimos-datos-disponibles/.

10. Benavides FG, Velarde JM, López-Ruiz M, Rodrigo F. Una década de éxito en la prevención de las lesiones por accidentes de trabajo en España. Seguridad y Salud en el Trabajo. 2011; 62:22-7.

11. Benavides FG, Benach J, Martínez JM, Gonzalez S. Description of fatal occupational injury rates in five selected European Union countries: Austria, Finland, France, Spain and Sweden. Safety Science. 2005; 43: 497-502.

12. Bailer J, Stayner LT, Stout NA, Gilbert SJ. Trends in rates of occupational fatal injuries in the United States (1983-1992). Occup Environ Med. 1998; 55: 485-489. 13. Santamaría N, Catot N, Benavides FG. Tendencias temporales de las lesiones mortales (traumáticas) por accidentes de trabajo en España (1992-2002), Gac. Sanit. 2006; 20:280-6.

14. Loomis D, Bena JF, Bailer AJ. Diversity of trends in occupational injury mortality in the United States, 1980-96. Injury Prevention. 2003; 9:9-14.

15. López-Ruiz M., Martínez J.M., Pérez K., Novoa A.M., Tobías A., Benavides F.G. Impact of road safety interventions on traffic-related occupational injuries in Spain, 2004-2010. Accident Analysis and Prevention. 2014; 66:114-9.

16. Dirección Nacional de la propiedad del automotor y de créditos prendarios. Evolución del parque automotor en la República Argentina 1966-1998 [en línea]. Argentina [citado 01 Sep 2018]. Disponible en: http://www.dnrpa.gov.ar/portal_ dnrpa/estadisticas/bolesta1/boletin0102/primera_seccion/pagina09.htm.

17. Dirección General de Estadísticas y Censos. El mercado laboral porteño y su relación con el conurbano [página principal en internet]. Argentina, septiembre de 2013, informe de resultado 573 [citado 01 Sep 2018]. Disponible en: http://www. estadisticaciudad.gob.ar/eyc/wp-content/uploads/2015/04/ir_2013_573.pdf.

18. Serra C, Ramada JM, Delclòs J, Benavides FG. Enfermedades Profesionales atendidas en el Parc de Salut Mar. 2015; 146:506-510.

19. Stocks J McNamee R van der Molen HF et al. Trends in incidence of occupational asthma, contact dermatitis, noise-induced hearing loss, carpal tunnel syndrome and upper limb musculoskeletal disorders in European countries from 2000 to 2012. Occup Env Med 2015;72:294-303.

20. Instituto Nacional de Estadística y Censo (INDEC). Trabajo e Ingresos, Mercado de Trabajo, Tasas e Indicadores Socioeconómicos (EPH) [página principal en internet]. Argentina, 2018, Vol. 2, No4. ISSN 2545-6636 [citado 01 Sep 2018]. Disponible en: https://www.indec.gov.ar/uploads/informesdeprensa/mercado_ trabajo_eph_1trim18.pdf. 